\title{
Impact of Anisotropy on Antiferromagnet Rotation in Heusler-type Ferromagnet/Antiferromagnet Epitaxial Bilayers
}

\author{
T. Hajiri, ${ }^{1, \text { * }}$ M. Matsushita, ${ }^{1}$ Y. Z. Ni, ${ }^{1}$ and H. Asano ${ }^{1}$ \\ ${ }^{1}$ Department of Crystalline Materials Science, Nagoya University, Nagoya 464-8603, Japan
}

(Dated: September 24, 2018)

\begin{abstract}
We report the magnetotransport properties of ferromagnet (FM)/antiferromagnet (AFM) $\mathrm{Fe}_{2} \mathrm{CrSi} / \mathrm{Ru}_{2} \mathrm{MnGe}$ epitaxial bilayers using current-in-plane configurations. Above the critical thickness of the $\mathrm{Ru}_{2} \mathrm{MnGe}$ layer to induce exchange bias, symmetric and asymmetric curves were observed in response to the direction of FM magnetocrystalline anisotropy. Because each magnetoresistance curve showed full and partial AFM rotation, the magnetoresistance curves imply the impact of the $\mathrm{Fe}_{2} \mathrm{CrSi}$ magnetocrystalline anisotropy to govern the AFM rotation. The maximum magnitude of the angular-dependent resistance-change ratio of the bilayers is more than an order of magnitude larger than that of single-layer $\mathrm{Fe}_{2} \mathrm{CrSi}$ films, resulting from the reorientation of AFM spins via the FM rotation. These results highlight the essential role of controlling the AFM rotation and reveal a facile approach to detect the AFM moment even in current-in-plane configurations in FM/AFM bilayers.
\end{abstract}

\section{INTRODUCTION}

Antiferromagnets (AFMs) show great potential to replace ferromagnets (FMs) in spintronic applications [13]. Compared with FMs, AFMs have the advantages of much faster spin dynamics [4, 5], more stability against charge and external field perturbations [6], and no stray field [7, 8]. However, since the AFM spins align in alternating directions of magnetic moments on individual atoms, the resulting zero net magnetization makes hard to control AFM magnetic moments. Recently, there have been several reports regarding the control of AFM moments by applying an electronic current in AFM films [9] and FM/AFM bilayers [10, 11], by field cooling (FC) 12, 13] and by applying an external field via the exchange-spring effect 14 17]. These studies demonstrated that the AFM moments can be controlled and detected using electronic transport measurements without the need for large-scale facilities such as synchrotron and neutron facilities [18].

AFM rotation is of interest because a more than $100 \%$ spin-valve-like signal has been achieved in tunneling anisotropic magnetoresistance (TAMR) stacks by controlling the AFM spin configuration via the exchangespring effect of FM on AFM [15]. The exchange coupling has been widely used in spintronic devices such as spin-valve-type magnetic memory devices to pin the FM magnetization [19, 20]. In contrast, TAMR utilizes the rotating AFM exchange-coupled to FM [14, 21, 22]. The rotating AFM can be linked to the shift of hysteresis loops (exchange bias) and broadening of the coercivity in magnetization measurements [18]. Although several studies have been reported regarding the rotating AFM [15, 18, 23, 24], almost all of the studies have been performed on polycrystalline stacks using AFM for IrMn. Since the AFM moments rotate with exchange-coupled FM, the AFM rotation behavior is expected to be affected by the FM magnetization switching process. Thus, the effect of FM magnetocrystalline anisotropy resulting from the full epitaxial growth is more interesting. In addition, all the studies have been performed using typical $3 d$ metal FMs such as NiFe and Co. Similar to successful studies on giant magnetoresistance (GMR) and tunneling magnetoresistance (TMR) [25, 26], there is a clear need to study high-quality heterostructures with more advanced compounds such as those with high-spin polarization needed for spintronic devices [27].

For the advanced materials, we focused on the Heusler compound $\mathrm{Fe}_{2} \mathrm{CrSi}$ (FCS), which is theoretically expected to be half-metallic FM [28]. The four-fold magnetocrystalline anisotropy constant of FCS is known to be $266 \mathrm{~J} / \mathrm{m}^{3}$ 29]. In addition, since only fully epitaxial stacks effectively provide the advanced properties, the Heusler compound $\mathrm{Ru}_{2} \mathrm{MnGe}$ (RMG) was selected for AFM; we succeeded in growing fully epitaxial FCS/RMG bilayers 30]. RMG exhibits the highest Néel temperature, $T_{N}=353 \mathrm{~K}$, among Heusler compounds [31]. In addition, RMG has a nearly half-metallic electronic structure [32]. Since the TAMR depends on spin configuration of AFM, such an AFM electronic structure is interesting.

In this letter, we systematically studied the magnetic and magnetotransport properties of FCS/RMG bilayers using current-in-plane (CIP) configurations. The angular-dependent resistance change $(\Delta R)$ ratio and exchange bias $\left(H_{e x}\right)$ exhibit a similar RMG thickness $\left(t_{\mathrm{RMG}}\right)$ dependence, indicating that RMG spin reorientation via FCS rotation is dominant in $\Delta R$ above a critical thickness $\left(t_{c}\right)$ to induce $H_{e x}$ even in CIP configurations. Above $t_{c}$, the magnetoresistance (MR) curves along the hard axes of FCS magnetocrystalline anisotropy exhibit a full rotation of AFM moments. However, a partial rotation of AFM moments is observed along the easy axes, demonstrating the effect of the FM magnetization reversal process on AFM rotation. Although previous studies have used current-perpendicular-to-plane configurations, 
these results indicate that CIP magnetotransport measurements in FM/AFM bilayer provide a facile approach to detect AFM moments and promote their application in AFM spintronics.

\section{EXPERIMENTAL DETAILS}

RMG/FCS bilayers were deposited on $\mathrm{MgO}$ (001) substrates by DC magnetron sputtering at a base pressure of approximately $5 \times 10^{-8}$ Torr. RMG thin films were deposited at a substrate temperature of $T_{s}=500{ }^{\circ} \mathrm{C}$ and then cooled to room temperature. Next, FCS was deposited on RMG at room temperature. After the FCS was deposited, the FCS/RMG bilayers were annealed at $500{ }^{\circ} \mathrm{C}$ for 30 minutes to achieve $L 2_{1}$ ordering of the FCS. In addition, we also deposited FCS at $T_{s}=500{ }^{\circ} \mathrm{C}$. The results were the same in both cases. The crystal structure was analyzed using both in-plane and out-of-plane $\mathrm{X}$-ray diffraction (XRD) measurements with $\mathrm{Cu} K \alpha$ radiation. The magnetic properties were characterized using vibrating sample magnetometry and superconducting quantum interference device (SQUID) magnetometry. The magnetotransport measurements were performed using the standard DC four-terminal method in the CIP configuration. To induce exchange coupling, the bilayers were annealed at $350 \mathrm{~K}$ for the SQUID measurements and at $375 \mathrm{~K}$ for the magnetotransport measurements for 30 minutes with applying field of $+10 \mathrm{kOe}$, and then cooled to $T=4 \mathrm{~K}$ with applying field of $+10 \mathrm{kOe}[33]$.

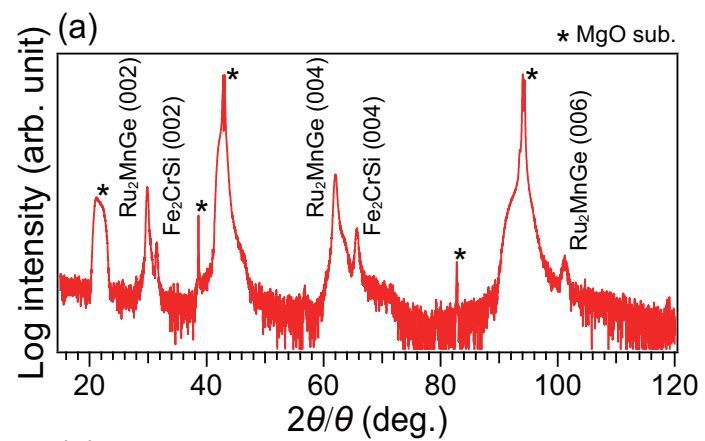

(b)

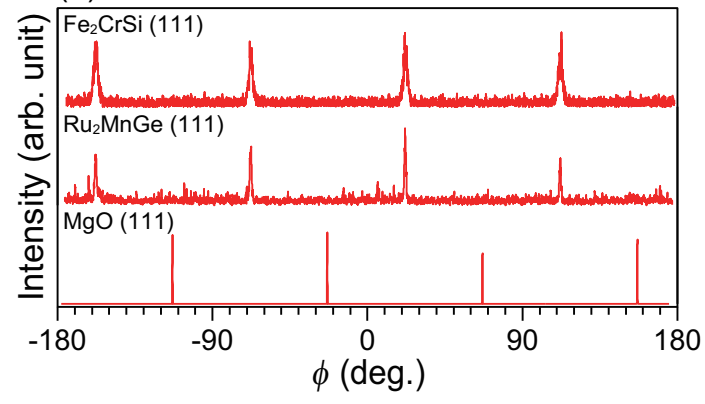

FIG. 1. (Color online) Out-of-plane $2 \theta / \theta$ scans (a) and inplane $\phi$-scans of FCS/RMG bilayers.

\section{RESULTS AND DISCUSSIONS}

XRD patterns of the FCS/RMG bilayers are presented in Fig. 1(a) and 1(b). As observed in Fig. 1(a), only the (00l) FCS and (00l) RMG peak series show Bragg peaks in the out-of-plane XRD pattern. Epitaxial growth is confirmed by the in-plane $\phi$-scans presented in Fig. 1(b). Both RMG (111) and FCS (111) peaks are observed with shifts of $45^{\circ}$ relative to the $\mathrm{MgO}$ (111) peaks. These results indicate that their epitaxial relationship is $\mathrm{FCS}(001)[100] / / \mathrm{RMG}(001)[100] / / \mathrm{MgO}(001)[110]$. In addition, since (111) reflection peaks of the Heusler alloy originate from superlattice reflections in the $L 2_{1}$ ordered structure [34], these XRD results indicate that high-quality FCS/RMG bilayers were obtained.

Figures 2(a)-2(c) shows the magnetic hysteresis loops of FCS $(5 \mathrm{~nm}) /$ RMG $\left(t_{\mathrm{RMG}} \mathrm{nm}\right)$ bilayers measured at $T=4 \mathrm{~K}$ after FC. The measurements were performed along the easy axis of FCS $\langle 100\rangle$. The $t_{\mathrm{RMG}}=5$ and $15 \mathrm{~nm}$ bilayers exhibit narrow hysteresis loops with a coercive field $H_{c}$ of approximately $100 \mathrm{Oe}$, which is similar to single-layer FCS films. The $t_{\mathrm{RMG}}=20 \mathrm{~nm}$ bilayers exhibited a much wider hysteresis loop with $H_{c}$ of approximately 860 Oe. On the other hand, the $t_{\mathrm{RMG}}=5 \mathrm{~nm}$ bilayer exhibited no hysteresis loop shift, whereas the $t_{\mathrm{RMG}}=15$ and $20 \mathrm{~nm}$ bilayers exhibited $H_{e x}=32$ and 155 Oe, respectively. The $t_{\mathrm{RMG}}$-dependent $H_{c}$ and $H_{e x}$ results are summarized in Fig. 2(d) at $T=4 \mathrm{~K} . H_{e x}$ is confirmed at above $t_{\mathrm{RMG}}=10 \mathrm{~nm}$, indicating that $t_{c}$ is between $t_{\mathrm{RMG}}=5$ and $10 \mathrm{~nm}$. A maximum $H_{e x}$ appears at $t_{\mathrm{RMG}}=20 \mathrm{~nm}$; then, $H_{\text {ex }}$ decreases with increasing $t_{\mathrm{RMG}}$. The same $t_{\mathrm{RMG}}$ thick-
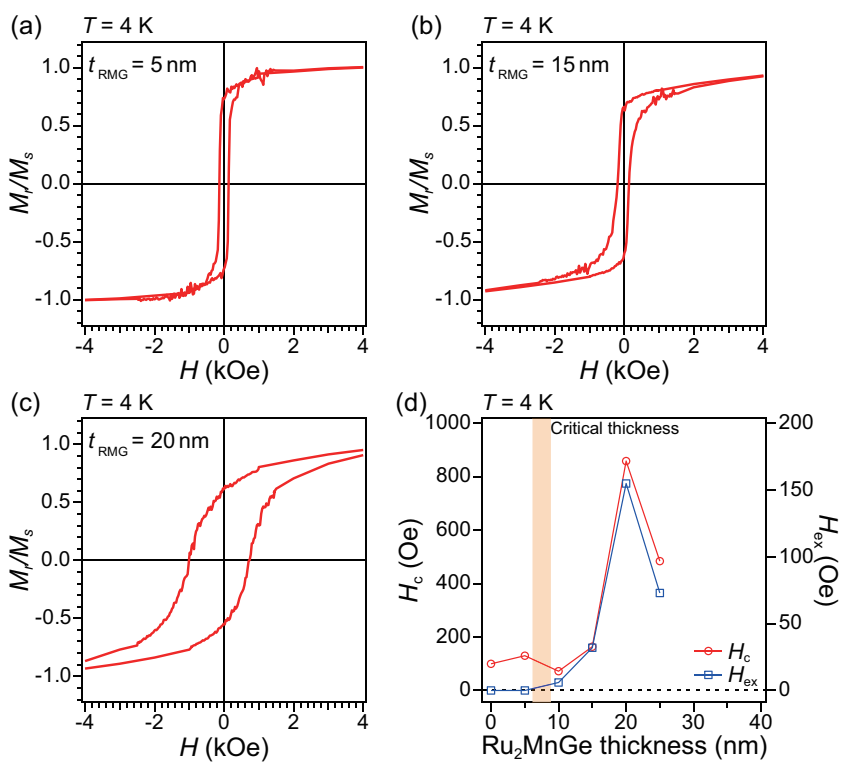

FIG. 2. (Color online) (a)-(c) Magnetic hysteresis loops of $t_{\mathrm{RMG}}=5,15$ and $20 \mathrm{~nm}$ at $T=4 \mathrm{~K}$ after $\mathrm{FC}$, respectively. (d) $H_{c}$ and $H_{e x}$ as a function of $t_{\mathrm{RMG}}$ at $T=4 \mathrm{~K}$. 

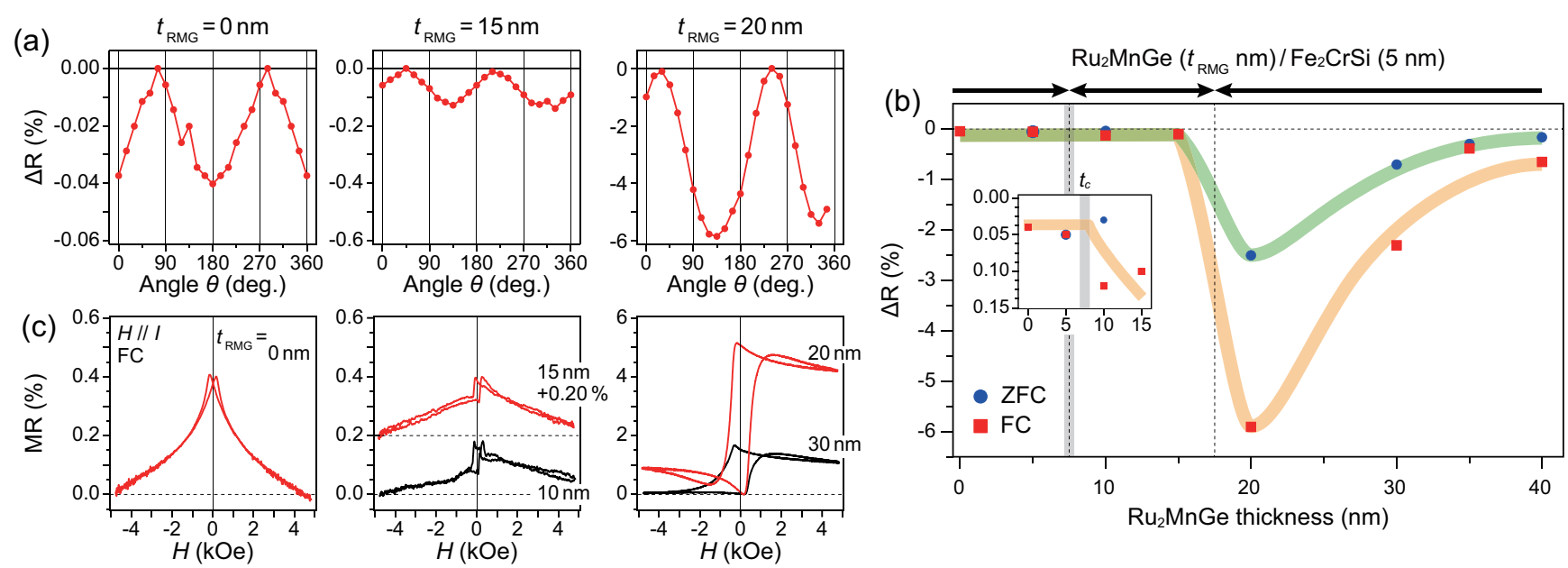

FIG. 3. (Color online) (a) $\Delta R$ as a function of the angle between the current and field for single-layer FCS films and FCS $(5 \mathrm{~nm}) / \mathrm{RMG}\left(t_{\mathrm{RMG}} \mathrm{nm}\right)$ bilayers. The measurements were performed at $T=4 \mathrm{~K}$ with an applied field of $+4 \mathrm{kOe}$. (b) $\Delta R$ as a function of $t_{\mathrm{RMG}}$ after both ZFC and FC at $T=4 \mathrm{~K}$. (c) MR curves as a function of applied field on single-layer FCS films and FCS $(5 \mathrm{~nm}) / \mathrm{RMG}\left(t_{\mathrm{RMG}} \mathrm{nm}\right)$ bilayers at $T=4 \mathrm{~K}$.

ness dependence was observed at $T=77 \mathrm{~K}$ for a wider $t_{\mathrm{RMG}}$ range [35]. $H_{c}$ shows no significant thickness dependence below $t_{\mathrm{RMG}}=15 \mathrm{~nm}$; then a jump is observed at $t_{\mathrm{RMG}}=20 \mathrm{~nm}$. However, $t_{\mathrm{RMG}}=20 \mathrm{~nm}$ shows much larger $H_{c}$ and $H_{e x}$ than $t_{\mathrm{RMG}}=25 \mathrm{~nm}$ at $T=4 \mathrm{~K}$, of which results are unusual behavior. The possible reason will be discussed later.

Next, we focused on the $t_{\mathrm{RMG}}$-dependent magnetotransport properties using CIP configuration. Figure 3(a) plots the $\Delta R$ ratio as a function of the relative angle $\theta$ between the current and FM magnetization direction of $\mathrm{FCS}(5 \mathrm{~nm}) / \mathrm{RMG}\left(t_{\mathrm{RMG}} \mathrm{nm}\right)$ bilayers at $T=4 \mathrm{~K}$ under an applied field of $+4 \mathrm{kOe}$ after FC. At $t_{\mathrm{RMG}}=0 \mathrm{~nm}$, a typical anisotropic magnetoresistance (AMR) ratio is confirmed with a negative value of approximately $-0.04 \%$. The negative AMR sign may originate from the half-metallic electronic structure of FCS, as discussed in recent theoretical and experimental studies [36, 37]. At $t_{\mathrm{RMG}}=15 \mathrm{~nm}$, the amplitude of $\Delta R$ increases, and its angular dependence shifts by approximately $45^{\circ}$. At $t_{\mathrm{RMG}}=20 \mathrm{~nm}$, the amplitude of $\Delta R$ is more than an order of magnitude larger than that of single-layer FCS films.

The $\Delta R$ ratios with respect to $t_{\mathrm{RMG}}$ at $T=4 \mathrm{~K}$ after both zero-field cooling (ZFC) and FC are summarized in Fig. 3(b). After FC, the $\Delta R$ ratios are independent of $t_{\mathrm{RMG}}$ below $t_{c}$. Above $t_{c}$, the $\Delta R$ ratios increase at 10 and $15 \mathrm{~nm}$. At $t_{\mathrm{RMG}}=20 \mathrm{~nm}$, the $\Delta R$ ratio drastically increases and then decreases upon further increasing $t_{\mathrm{RMG}}$. These $t_{\mathrm{RMG}}$-dependent $\Delta R$ ratios are similar to the exchange bias, as observed in Fig. 2(d). Moreover, as observed in Fig. 3(b), the $\Delta R$ ratios are enlarged by FC, demonstrating the effect of exchange coupling on the $\Delta R$ ratio. Note that since the exchange coupling might exist even without $\mathrm{FC}$ [38, 39], the $\Delta R$ ratio after ZFC is larger than that for single-layer FCS films.

In addition, a relationship is observed between the $\Delta R$ ratios, the shape of the MR curves and exchange bias. The MR curves measured as a function of the applied magnetic field are presented in Fig. 33(c). The MR curves of the single-layer FCS films $\left(t_{\mathrm{RMG}}=0 \mathrm{~nm}[40]\right)$ ) are symmetric, which originates from the FCS AMR. In contrast, above $t_{c}$, the curves of the bilayers differ from typical AMR curves. The $t_{\mathrm{RMG}}=10$ and $15 \mathrm{~nm}$ bilayers exhibit small asymmetric curves, and the asymmetry increases at $t_{\mathrm{RMG}}=20$ and $30 \mathrm{~nm}$. According to previous FM/AFM studies [15, 18, 24], the asymmetric MR curves originate from the partial rotation of the AFM moments due to the applied external field via the FM rotation, whereas the symmetric MR curves originate from the full rotation of the AFM moments.

Finally, we would like to discuss the origin of the anomalous magnetic properties of the bilayers. Figures 4(a) and 4(b) compare different measurement conditions; the sensing current $I$ was applied in directions parallel to FCS/RMG [100] and [1-10], respectively. As observed in Fig. 4(a) for $I \|$ [100], asymmetric MR curves are obtained along $H \|[100]\left(\theta=0^{\circ}\right)$ and [010] $\left(\theta=90^{\circ}\right)$. On the other hand, symmetric MR curves are obtained along $H \|[110]\left(\theta=45^{\circ}\right)$ and [-110] $\left(\theta=135^{\circ}\right)$. For $I \|[1-10]$, the symmetric and asymmetric relations with respect to crystalline direction do not change; symmetric MR curves are obtained along $H \|[1-10]\left(\theta=0^{\circ}\right)$ and [110] $\left(\theta=90^{\circ}\right)$, and asymmetric MR curves are obtained along $H \|[100]\left(\theta=45^{\circ}\right)$ and $[0-10]\left(\theta=-45^{\circ}\right)$. Although FC-direction dependence was performed, no change was observed. These results indicate that the symmetric and asymmetric curves are not determined by 

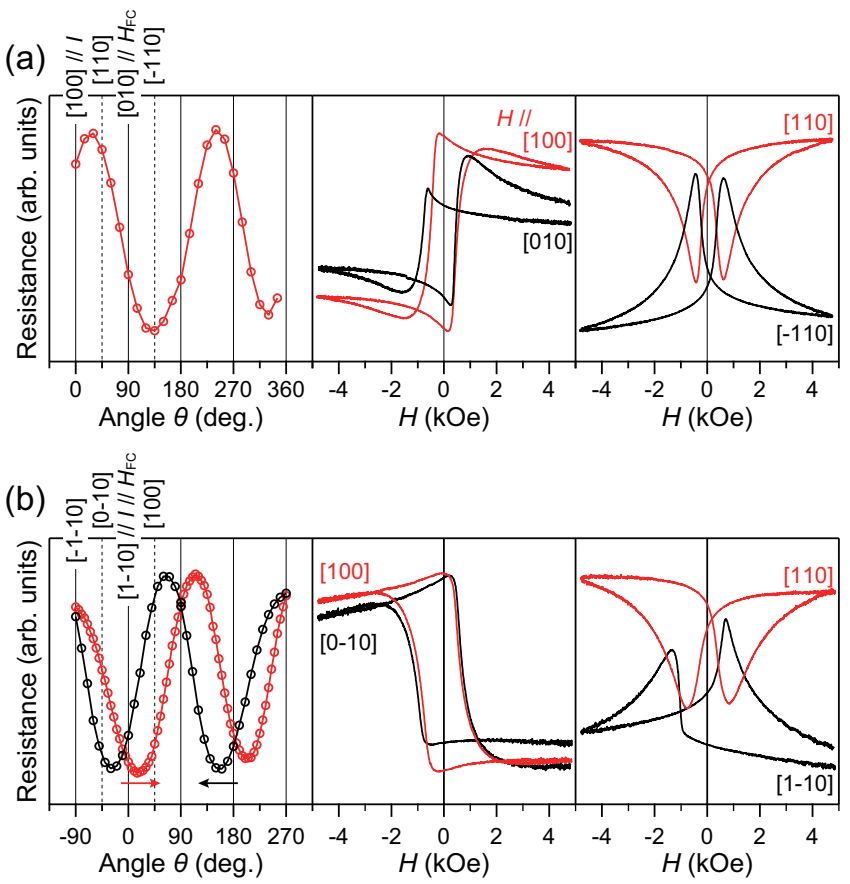

(c)

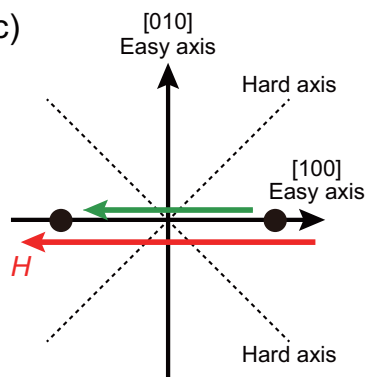

(d)

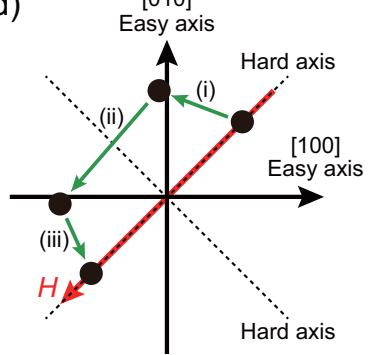

FIG. 4. (Color online) (a, b) Magnetotransport properties of the configurations $I \|[100]$ and $\|[1-10]$, respectively. (c, d) FCS magnetization switching process where the field sweeps along the easy and hard axes, respectively.

the relative angle $\theta$ between $H$ and $I$, indicating that the obtained angular dependences are not typical AMR of FM. Then, because the symmetric and asymmetric relations are not changed by the FC directions, the factor governing the angular dependence of the bilayers is not the sensing current or FC directions but the FCS/RMG crystalline direction.

One possible cause of the crystalline-directiondependent AFM rotation is the FM magnetization switching process. FCS has four-fold magnetocrystalline anisotropy, where the easy axes are oriented along $\langle 100\rangle$ and $\langle 010\rangle$, and the hard axes are oriented along $\langle 110\rangle$ and $\langle 1-10\rangle[29]$. This property indicates that symmetric MR curves appear along the hard axes of FCS, and asymmetric MR curves appear along the easy axes of FCS. It is well known that the FM magnetization switching processes with four-fold magnetocrystalline anisotropy differ along the easy and hard axes. As presented in Fig. 廿4(c), the magnetization rotates by $180^{\circ}$ along the easy axes.
On the other hand, as presented in Fig. 4(d), the magnetization rotates in 3 steps along the hard axes; (i) the magnetization rotates toward the nearest easy axis, (ii) the magnetization jumps in a direction close to the other easy axis, and (iii) the magnetization finally rotates toward the applied field direction [41]. Therefore, the magnetization rotates by up to $90^{\circ}$ along the hard axis. A possible explanation for the full and partial AFM rotations along FCS/RMG $\langle 100\rangle$ and $\langle 110\rangle$ are that the AFM can fully follow the FM magnetization switching via the FM rotation when the field sweep is along the hard axis of FM because the magnetization rotates slightly (up to $90^{\circ}$ ). On the other hand, the AFM cannot fully follow when the field sweep is along the easy axis of FM because the magnetization rotates a lot $\left(180^{\circ}\right)$. These results could provide a route to understanding the AFM rotation behavior.

Since the MR curves suggest the importance of AFM spin configuration, as discussed above, the larger $\Delta R$ ratio compared with that of only single-layer FCS films can be considered to be due to AFM moments. To date, there have been several studies of AFM AMR due to spin flop [6], crystalline AMR originating from large anisotropies in the relativistic electronic structure [16] and AFM spin configurations with respect to current direction [12]. In the crystalline AMR study, the AFM spins were reoriented by applying magnetic fields via the exchange spring effect [14], which is the same condition as that in our study. In addition, the $\Delta R$ ratio was obtained under $H=4 \mathrm{kOe}$ with small angle steps $\left(\sim 15^{\circ}\right)$, indicating that the AFM moments can fully follow the FM magnetization rotation as mentioned above. The AFM spin reorientation is reinforced by the delay of angular dependence due to the exchange-spring effect 15], as shown in Fig. 4(b). Then, the resistance is higher for the configuration of AFM moments aligned along [110] and [-110] than for the configuration of AFM moments aligned along [1-10] and [-110]. These resistance changes due to the AFM spin direction have been reported in FM/AFM bilayers [16]. Moreover, the asymmetric MR curves were transformed into symmetric MR curves with increasing temperature. Therefore, we conclude that the obtained larger $\Delta R$ ratio compared with that of only single-layer FCS films originates from the reorientation of the AFM moments by applying magnetic fields via FM rotation. Then, the unusual $t_{\mathrm{RMG}}$-dependent $H_{e x}$ and $H_{c}$ might be caused by rotating AFM and/or exchange-spring effect. As mentioned in the introduction, rotating AFM can be linked to $H_{e x}$ and $H_{c}$ [18]. As similar to $H_{e x}$ and $H_{c}$, $t_{\mathrm{RMG}}=20 \mathrm{~nm}$ shows much larger $\Delta R$ ratio than that of $t_{\mathrm{RMG}}=30 \mathrm{~nm}$. Since $\Delta R$ ratio originates from the reorientation of the AFM moments via exchange-spring effect, these results might indicate the $t_{\mathrm{RMG}}$-dependent rotating AFM and/or exchange-spring effect. The obtained $\Delta R$ ratio of approximately $5.9 \%$ is much larger than other AFM AMR ratios for $\mathrm{Sr}_{2} \mathrm{IrO}_{4}$ of approximately $1 \%$ and 
MnTe of approximately $1.6 \%$. This result might be related to either the nearly half-metallic RMG electronic structure or the electronic structures of both RMG and FCS.

\section{CONCLUSION}

We performed a magnetotransport study of FCS/RMG bilayers to clarify the AFM rotation behavior. In addition to the same $t_{\mathrm{RMG}}$ thickness dependence of the magnitude of the $\Delta R$ ratio and exchange bias, the MR curves changed from symmetric to asymmetric based on $t_{\mathrm{RMG}}$ in response to the direction of FCS magnetocrystalline anisotroy. The maximum $\Delta R$ ratio of the bilayers was more than an order of magnitude larger than that of single-layer FCS films due to the reorientation of AFM moments via the FM rotation. We also observed that the AFM moments could fully rotate when the field sweep was along the hard axes of FCS but could not fully rotate along the easy axes of FCS, demonstrating the impact of FCS magnetocrystalline anisotropy to govern the AFM rotation. These results provide profound insights into the control of the AFM moments and promote the application of AFM in spintronics.

\section{ACKNOWLEDGMENTS}

The authors gretefully acknowledge M. Kuwahara and K. Saitoh for their invaluable support. Part of this work was supported by the Japan Society for the Promotion of Science (JSPS) Program for Advancing Strategic International Networks to Accelerate the Circulation of Talented Researchers.

* Electronic mail: t.hajiri@numse.nagoya-u.ac.jp

[1] H. V. Gomonay and V. M. Loktev, Low Temp. Phys. 40, 17 (2014).

[2] T. Jungwirth, X. Martí, P. Wadley and J. Wunderlich, Nat. Nanotech. 11, 231 (2016).

[3] V. Baltz, A. Manchon, M. Tsoi, T. Moriyama, T. Ono, and Y. Tserkovnyak, arXiv: 1606.04284 (2016).

[4] J. Železný, H. Gao, K. Výborný, J. Zemen, J. Mašek, A. Manchon, J. Wunderlich, J. Sinova, and T. Jungwirth, Phys. Rev. Lett. 113, 157201 (2014).

[5] A. V. Kimel, A. Kirilyuk, A. Tsvetkov, R. V Pisarev, and T. Rasing, Nature 429, 850 (2004).

[6] D. Kriegner, K. Výborný, K. Olejník, H. Reichlová, V. Novák, X. Martí, J. Gazquez, V. Saidl, P. Nĕmec, V. V. Volobuev, G. Springholz, V. Holý, and T. Jungwirth, Nat. Commun. 7, 11623 (2016).

[7] S. Loth, S. Baumann, C. P. Lutz, D. M. Eigler, and A. J. Heinrich, Science 335, 196 (2012).

[8] D. Petti, E. Albisetti, H. Reichlová, J. Gazquez, M. Varela, M. Molina-Ruiz, A. F. Lopeandía, K. Olejník,
V. Novák, I. Fina, B. Dkhil, J. Hayakawa, X. Martí, J. Wunderlich, T. Jungwirth, and R. Bertacco, Appl. Phys. Lett. 102, 192404 (2013).

[9] P. Wadley, B. Howells, J. Železný, C. Andrews, V. Hills, R. P. Campion, V. Novák, K. Olejnik, F. Maccherozzi, S. S. Dhesi, S. Y. Martin, T. Wagner, J. Wunderlich, F. Freimuth, Y. Mokrousov, J. Kuneš, J. S. Chauhan, M. J. Grzybowski, A. W. Rushforth, K. W. Edmonds, B. L. Gallagher, and T. Jungwirth, Science 351, 587 (2016).

[10] H. Sakakibara, H. Ando, Y. Kuroki, S. Kawai, K. Ueda, and H. Asano, J. Appl. Phys. 117, 17D725 (2015).

[11] X.-L. Tang, H.-W. Zhang, H. Su, Z.-Y. Zhong, and Y.-L. Jing, Appl. Phys. Lett. 91, 122504 (2007).

[12] X. Martí, I. Fina, C. Frontera, J. Liu, P. Wadley, Q. He, R. J. Paull, J. D. Clarkson, J. Kudrnovský, I. Turek, J. Kuneš, D. Yi, J.-H. Chu, C. T. Nelson, L. You, E. Arenholz, S. Salahuddin, J. Fontcuberta, T. Jungwirth, and R. Ramesh, Nat. Mater. 13, 367 (2014).

[13] D. Petti, E. Albisetti, H. Reichlová, J. Gazquez, M. Varela, M. Molina-Ruiz, A. F. Lopeandía, K. Olejník, V. Novák, I. Fina, B. Dkhil, J. Hayakawa, X. Martí, J. Wunderlich, T. Jungwirth, and R. Bertacco, Appl. Phys. Lett. 102, 192404 (2013).

[14] A. Scholl, M. Liberati, E. Arenholz, H. Ohldag, and J. Stöhr, Phys. Rev. Lett. 92, 247201 (2004).

[15] B. G. Park, J. Wunderlich, X. Martí, V. Holý, Y. Kurosaki, M. Yamada, H. Yamamoto, A. Nishide, J. Hayakawa, H. Takahashi, A. B. Shick, and T. Jungwirth, Nat. Mater. 10, 347 (2011).

[16] I. Fina, X. Martí, D. Yi, J. Liu, J. H. Chu, C. RayanSerrao, S. Suresha, A. B. Shick, J. Železný, T. Jungwirth, J. Fontcuberta, and R. Ramesh, Nat. Commun. 5, 4671 (2014).

[17] R. Galceran, I. Fina, J. Cisneros-Fernández, B. Bozzo, C. Frontera, L. López-Mir, H. Deniz, K. W. Park, B. G. Park, Ll. Balcells, X. Martí, T. Jungwirth and B. Martínez, Sci. Rep. 6, 35471 (2016).

[18] X. Martí, B. G. Park, J. Wunderlich, H. Reichlová, Y. Kurosaki, M. Yamada, H. Yamamoto, A. Nishide, J. Hayakawa, H. Takahashi, and T. Jungwirth, Phys. Rev. Lett. 108, 017201 (2012).

[19] S. A. Wolf, D. D. Awschalom, R. A. Buhrman, J. M. Daughton, S. von Molnár, M. L. Roukes, A. Y. Chtchelkanova, and D. M. Treger, Science 294, 1488 (2001).

[20] J. Åkerman, Science 308, 508 (2005).

[21] J. Geshev, L. G. Pereira, and J. E. Schmidt, Phys. Rev. B 66, 134432 (2002).

[22] J. Wu, J. S. Park, W. Kim, E. Arenholz, M. Liberati, A. Scholl, Y. Z. Wu, C. Hwang, and Z. Q. Qiu, Phys. Rev. Lett. 104, 217204 (2010).

[23] Y. Y. Wang, C. Song, B. Cui, G. Y. Wang, F. Zeng, and F. Pan, Phys. Rev. Lett. 109, 137201 (2012).

[24] X. Zhou, L. Ma, Z. Shi, W. J. Fan, R. F. L. Evans, J.-G. Zheng, R. W. Chantrell, S. Mangin, H. W. Zhang, and S. M. Zhou, Sci. Rep. 5, 9183 (2015).

[25] Y. Sakuraba, K. Izumi, T. Iwase, S. Bosu, K. Saito, K. Takanashi, Y. Miura, K. Futatsukawa, K. Abe, and M. Shirai, Phys. Rev. B 82, 094444 (2010).

[26] H. X. Liu, Y. Honda, T. Taira, K. I. Matsuda, M. Arita, T. Uemura, and M. Yamamoto, Appl. Phys. Lett. 101, 132418 (2012).

[27] M. Jourdan, J. Minár, J. Braun, A. Kronenberg, S. Chadov, B. Balke, A. Gloskovskii, M. Kolbe, H. J. 
Elmers, G. Schönhense, H. Ebert, C. Felser, and M. Kläui, Nat. Commun. 5, 3974 (2014).

[28] B. A. Hamad, Eur. Phys. J. B 80, 11 (2011).

[29] T. Miyawaki, M. Foerster, S. Finizio, C. A. F. Vaz, M.A. Mawass, K. Inagaki, N. Fukatani, L. Le Guyader, F. Nolting, K. Ueda, H. Asano, and M. Kläui, J. Appl. Phys. 114, 073905 (2013).

[30] N. Fukatani, K. Inagaki, T. Miyawaki, K. Ueda, and H. Asano, J. Appl. Phys. 113, 17C103 (2013).

[31] N. Fukatani, H. Fujita, T. Miyawaki, K. Ueda, and H. Asano, IEEE Trans. Magn. 48, 3211 (2012).

[32] S. Ishida, S. Kashiwagi, S. Fujii, and S. Asano, Physica B 210, 140 (1995).

[33] The magnetic heat treatments have been done under the maximum temperature of each equipment of the SQUID measurements and the magnetotransport measurements.

[34] Y. Sakuraba, J. Nakata, M. Oogane, H. Kubota, Y. Ando, A. Sakuma, and T. Miyazaki, Jpn. J. Appl. Phys. 44, 6535 (2005).

[35] See Supplemental Material at. for the exchange bias at $T=77 \mathrm{~K}$.

[36] S. Kokado, M. Tsunoda, K. Harigaya, and A. Sakuma, J. Phys. Soc. Japan 81, 024705 (2012).
[37] Y. Sakuraba, S. Kokado, Y. Hirayama, T. Furubayashi, H. Sukegawa, S. Li, Y. K. Takahashi, and K. Hono, Appl. Phys. Lett. 104, 172407 (2014).

[38] S. Anandakumar, V. S. Rani, S. Oh, and C. Kim, Thin Solid Films 519, 1020 (2010).

[39] S. Brück, J. Sort, V. Baltz, S. Suriñach, J. S. Muñoz, B. Dieny, M. D. Baró, and J. Nogués, Adv. Mater. 17, 2978 (2005).

[40] Since single-layer FCS (5 nm) films did not show clear MR curves, we used the MR curve of FCS (200 nm). On the other hand, since FCS shows thickness dependent AMR ratio, we used AMR curves of FCS (5 nm). The thickness dependent AMR ratio has been reported in single-layer epitaxial Fe films; M. Tondra, D. K. Lottis, K. T. Riggs, Y. Chen, E. D. Dahlberg, and G. A. Prinz, J. Appl. Phys. 73, 6393 (1993).

[41] T. Hajiri, T. Yoshida, S. Jaiswal, M. Filianina, B. Borie, H. Ando, H. Asano, H. Zabel, and M. Kläui, Phys. Rev. B 94, 184412 (2016). 